\title{
A universal automated complex for control and diagnostics of semiconductor devices and structures
}

\author{
R.V. Konakova, O.E. Rengevych, A.M. Kurakin, Ya.Ya. Kudryk \\ Institute of Semiconductor Physics, NAS Ukraine, 45 prospect Nauky, 03028 Kyiv, Ukraine \\ Phone: 38(044) 265 6182; fax: 38(044) 265 8342; e-mail: konakova@isp.kiev.ua
}

\begin{abstract}
We present a universal automated complex for control and diagnostics. It is intended to measure static, pulse and capacitance-voltage characteristics of two- and threeterminal networks, both at room temperature and in $77-1000 \mathrm{~K}$ temperature range. A distinguishing feature of complex construction is the possibility for simulation of interrelation between parameters of the objects studied. The complex has been tested when studying the effect of $\gamma$ - and microwave radiations on parameters of gallium arsenide SB-FETs, GaN-based HEMTs and silicon carbide SBDs.
\end{abstract}

Keywords: diagnostics of semiconductor devices, automation of measurements.

Paper received 31.07.02; accepted for publication 17.12.02.

\section{Introduction}

Present-day manufacturing of semiconductor element base is characterized by a wide range of products that differ in their specifications and applications. Manufacturing dependence on rapidly varying market situation requires new instrumentation for check and measurement to provide competitive ability. Such instrumentation should be sufficiently flexible for easy integration into the existing measuring complexes, as well as possess wide functional capabilities that would enable to modify complexes on user's demands [1].

Now at research laboratories, as well as in industry, instrumentation of Western production for check and diagnostics is widely used [2-4]. Among the most popular instruments are, for instance, such as automated curve tracers HP 4145 and HP 4155 whose range of currents (voltages) measured is $10^{-12}-10^{-1} \mathrm{~A}\left(10^{-3}-10^{2} \mathrm{~V}\right)$, the relative measurement error being no more than $0.5 \%$; precise LCR-meters HP 4284, Genrad 1689 whose ranges of resistances, inductances and capacitances measured are $10^{-2}-10^{5} \Omega, 10^{-8}-10^{5} \mathrm{H}$ and $10^{-14}-10^{-1} \mathrm{~F}$, respectively, the relative measurement error being no more than $0.2 \%$. At the moment these instruments meet the requirements imposed on the facilities of such class and provide high accuracy and reproducibility of the results of measurements. However, their high cost and relatively low operation speed (no more than 20 measurements per second [5]) are not optimal for their use in test rigs for express check of parameters and characteristics of semiconductor devices.

The domestic complexes for control and diagnostics are made on the basis of industrial curve tracers. They make it possible to take characteristics of semiconductor devices of practically all types over wide range of currents and voltages. However, their operation speed is low and, as a result, their use is costly $[6,7]$. Solution of this problem lies in development of domestic multifunctional automated complexes characterized by high operation speed and rather low cost.

Here we present the results of our activity in this line taking, as an example, development and fabrication of both hard- and software for a universal automated complex for control and diagnostics of semiconductor devices. This complex is a logic extension of the test rigs for check and diagnostics that have been developed and fabricated by us earlier [8-10].

\section{Construction and potentialities of the complex}

The block diagram of our complex for control and diagnostics is presented in Fig. 1. The complex is made on the 


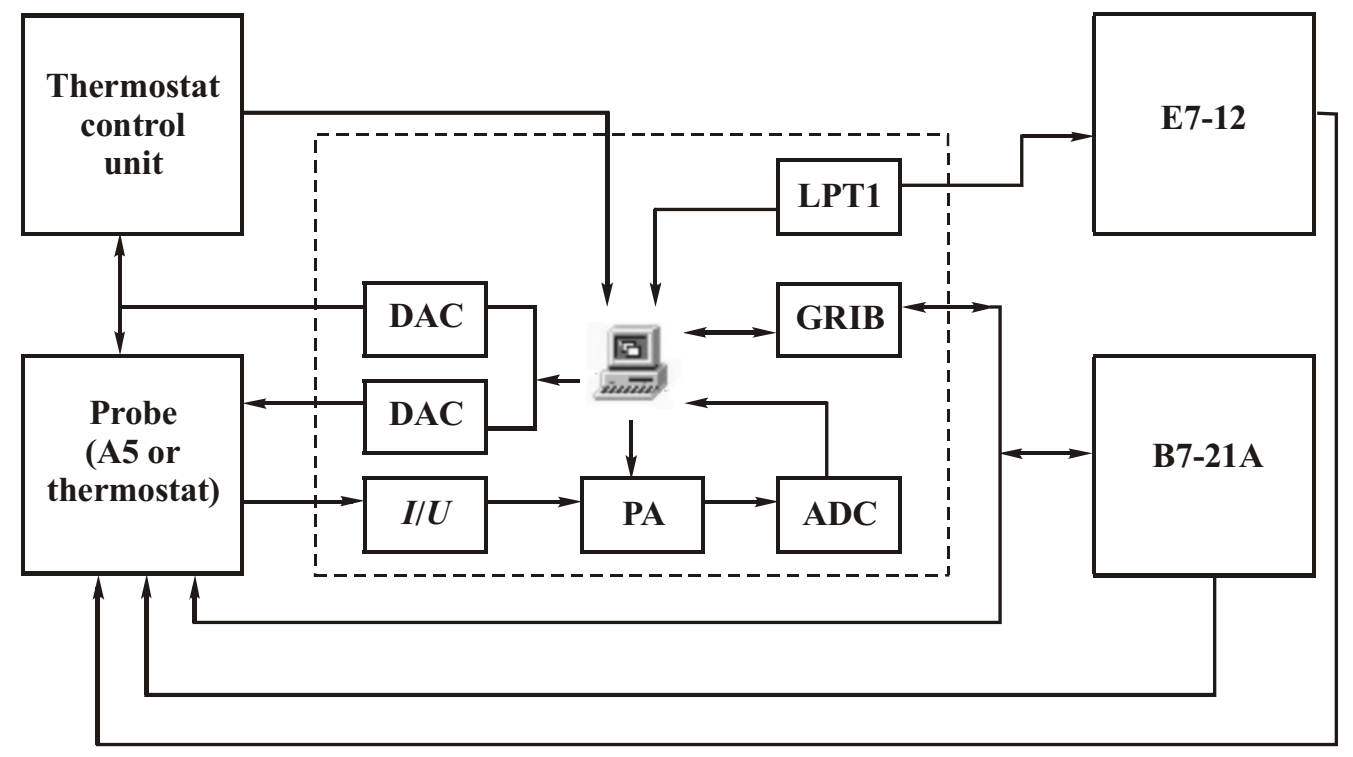

Fig. 1. Block diagram of the complex. ADC - analog-to-digital converter, DAC - digital-to-analog converter, GPIB - general purpose interface bus, $I / U$ - current-to-voltage converter, PA - programmable amplifier.

basis of IBM-compatible computer (486DX80). Its functional capabilities are as follows:

1) measurements of static $I-V$ curves for two- and three-terminal networks in the range of currents from $1 \times 10^{-11}$ to $2 \mathrm{~A}$ and voltages up to $50 \mathrm{~V}$ (two ranges: $0-10 \mathrm{~V}$ and $0-50 \mathrm{~V}$ ), the relative measurement error being no more than $0.5 \%$;

2) measurements of pulse $I-V$ curves for two- and three-terminal networks in the range of currents from $1 \times 10^{-4}$ to $2 \mathrm{~A}$ and voltages up to $10 \mathrm{~V}$ at pulse duration (programmable) from $10^{-6} \mathrm{~s}$ to the case of continuous current, the relative current measurement error being no more than $1 \%$;

3) measurements of $C$ - $V$ curves in the range of capacitances from $10^{-14}$ to $10^{-7} \mathrm{~F}$, the relative measurement error being no more than $0.2 \%$;

4) measurements of $C$ - $V$ curves and static $I-V$ curves in the $77-1000 \mathrm{~K}$ temperature range; load;

5) protection of sample tested against current over-

6) possibility to perform measurements for both discrete elements and elements on wafers;

7) possibility to perform simulation of interrelation between the parameters of objects studied, as well as complete automation of measurements (no operator attention is required during the measurement process for devices of the same batch);

8) high operation speed (to illustrate, a set of eight $I$ $V$ curves - 255 points for each - is measured for $5 \mathrm{~ms}$, i.e., an order faster than in [11]).

The complex was designed as a multifunctional facility of open architecture. Such an approach enabled us to make a flexibly adjustable system involving test rigs that complemented each other. They had a common computer and common software. Each of functional potentiali- ties of the complex can be realized independently (or with an incomplete set of functions). This fact may considerably reduce the complex cost.

The complex construction involves two boards built into computer (bus ISA), a voltmeter B7-21A, bridge E712 , thermostat, unit to control thermostat, contact facility (when the devices on a wafer are measured, this is an automated probe A5). A board that is built into the computer has two 12-bit digital-to-analog converters (DACs), a current-to-voltage converter, programmable amplifier, 12-bit analog-to-digital converter (ADC) and programmable timer, as well as a general purpose interface bus (GPIB) controller board. In this configuration the GPIB controller is used only to connect a bridge E7-12 for measurement of $C-V$ curves. However, application of the GPIB controller (that is a traditional interface in various modern devices, of domestic, as well as overseas, production) enables to extend the complex capabilities by connecting various devices.

One of distinguishing features of the complex is application of standard computer interfaces for data exchange with the peripheral facilities. To illustrate, a voltmeter B7-21A that is used when measuring static $I-V$ curves of two- and three-terminal networks is served by an interface "Centronics" (printer port LPT1). The data given by a device are read from numeric printer interface in binary-decimal code, multiplexed and transmitted to computer through interface "Centronics". Control over the automated probe $\mathrm{A} 5$ is also exerted through the printer port (LPT1). At a later time we plan to completely rule out the boards built into computer. Their functions will be realized on the basis of microcontrollers connected to computer through serial port. Such modification will made the complex still more universal and independent 


\section{R.V. Konakova et al.: A universal automated complex for control and ...}

of computer configuration and presence (or absence) of bus ISA or PCI.

Setting of a required temperature value in the thermostat can be made by a signal from computer, as well as manually. Control from computer is made by applying a digital or analog signal (applying a corresponding voltage from DAC). When hand-operating, control is exerting by setting a code that is proportional to temperature. Processes of temperature establishment are regulated by the microcontroller in the thermostat control unit. Feedback with computer is made only when a ready signal comes from the thermostat. The temperature setting error in the $77-1000 \mathrm{~K}$ range is $\pm 0.5 \mathrm{~K}$. Stability of temperature maintenance is $0.1 \mathrm{~K}$; this is twice as good as that provided by the technique proposed in [12].

Application of a miniature Schottky-barrier (SB) diode as a temperature sensor demonstrating linear temperature dependence of voltage (at a constant value of diode current) over the whole operating range made it possible to rule out a compensation thermocouple. (One of its junctions had to be at a constant temperature, or this temperature had to be uninterruptedly monitoredsay, with a semiconductor sensor, as it was made in [13].)

To provide the most complete use of complex potentialities, we have developed common software. This made it possible not only to exert efficient control over the system, but also to perform simulation of interrelation between parameters. From the results of measurements the following characteristics are calculated: saturation current, transconductance, cutoff voltage, channel and contact resistances - for field-effect transistors (FETs); Schottky barrier height, ideality factor, saturation current and series resistance - for diodes; temperature dependence of parameters and their distribution over wafer. When studying the effect of external factors (radiation, microwave field, thermal annealing, ultrasound, etc.), the complex enables one to obtain the database for the above parameters and perform analysis of their dependence on these factors. Software (common for the whole complex) makes it possible to perform simulation of interrelations between the parameters of the objects studied.

The complex has been tested when studying the effect of $\gamma$-and microwave radiation on the parameters of lownoise gallium arsenide SB-FETs and test pieces of FETs, as well as high electron mobility transistors (HEMTs) based on GaAs [14] and GaN. The device characteristics were measured in the pulse mode. This made it possible to practically completely exclude the effect of device structure heating. Shown in Fig. 2 are $I-V$ curves taken in the pulse (full curve) and static (dashed curve) modes for the same transistor. One can see that at high drain-source voltages a portion of $I-V$ curve with negative differential resistance is observed in the static mode. This results from structure overheating with current. It should be noted that such pattern is observed even with allowance made for the fact that time of measurement for the whole set of $I-V$ curves (when the sample studied under load) is from 1 to $2 \mathrm{~s}$, depending on the intervals between consecutive gatesource and drain-source voltage values.
The operation parameters of HEMTs found from the experimental $I$ - $V$ curves (saturation current, transconductance, cutoff voltage) and characteristic resistance demonstrated high reproducibility. Shown in Fig. 3 are typical $C$ - $V$ curves for $\mathrm{SiC}$-based diodes (a) and typical temperature dependence of $I-V$ curves for $\mathrm{SiC}$-based diode.

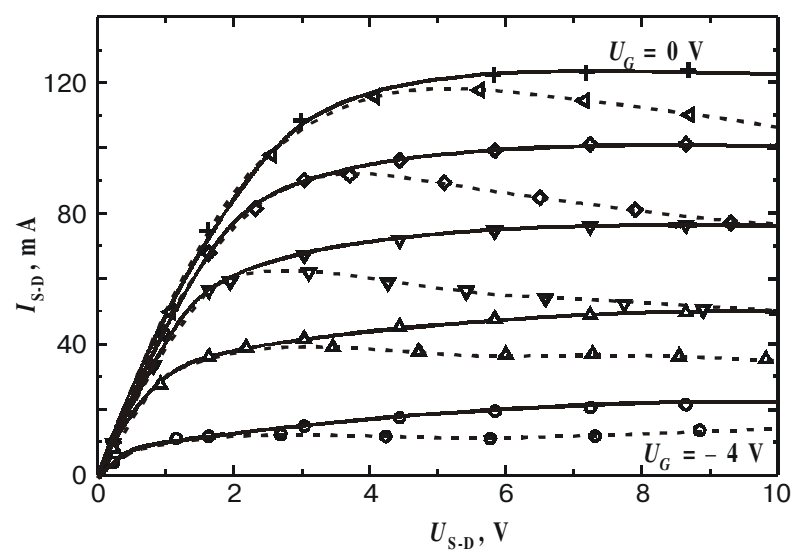

Fig. 2. $I-V$ curves for a GaN-based transistor taken in the pulse (full curve) and static (dashed curve) modes.
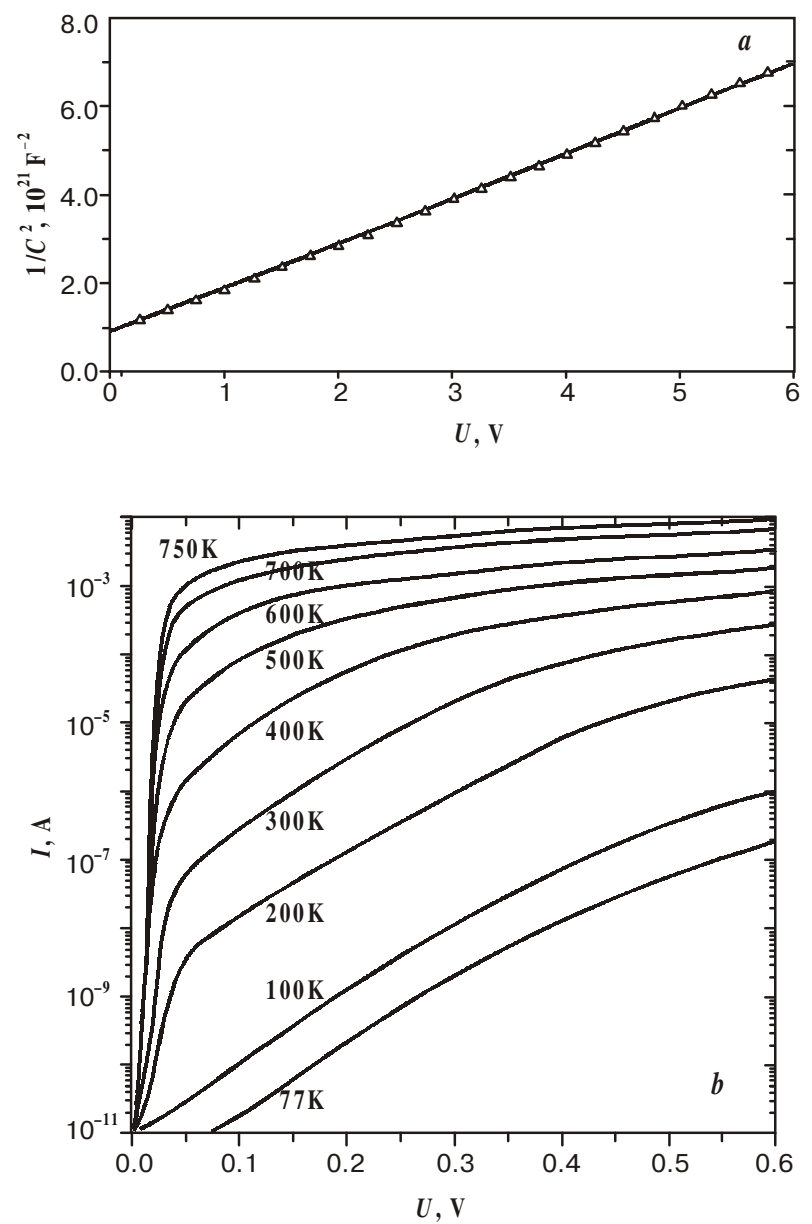

Fig. 3. Typical $C$ - $V$ curves for SiC-based diodes $(a)$ and typical temperature dependence of $I-V$ curves for SiC-based diodes $(b)$. 


\section{Conclusion}

We have developed and tested a universal automated complex for control and diagnostics. It enables to measure static, pulse, $C$ - $V$ curves and temperature characteristics of two- and three-terminal networks, as well as process the results obtained (performing calculation operations during measurements), present and save information in a form convenient for operator.

Such complex features, as multifunctionality, universality, small size, high operation speed, possibility to extend and modify its functional potentialities, enable one to use this complex as part of various plants for control and diagnostics, both for researches and in industry, to check device parameters and reject potentially unreliable devices at the output inspection stage.

\section{References}

1. R.V. Konakova, Yu.A. Tkhorik, V.I. Faynberg, E.A. Soloviev, F. Štofanik, Diagnostics of semiconductor devices from their electric, photoelectric and pulse characteristics using the complex "Capron" // Optoelektronika i Poluprovodnikovaya Tekhnika No 18, pp. 35-46. (1990) (in Russian).

2. L.S. Yu, D. Qiao, L. Jia, S.S.Lau, Y. Qi, K.M. Lau, Study of Schottky barrier of Ni on p-GaN // Appl. Phys. Lett. 79(27), pp. 4536-4538 (2001)

3. Q. Chen, J.W. Yang, M. Blasingame, C. Faber, A.T. Ping, I. Adesida, Microwave electronics device applications of AlGaN/ GaN heterostructures // Mater. Sci. \& Eng. B59, pp. 395-400 (1999).

4. C.A. Dimitriadis, Th. Karakostas, S. Logothetidis, G. Kamarinos, J. Brini, G. Nouet, Contacts of titanium nitride to $n$ - and p-type gallium nitride films // Solid-St. Electron. $\mathbf{4 3}$, pp. 1969-1972 (1999).
5. Advanced Test Equipment // www.atecorp.com

6. V.N. Petrov, M.N. Petrov, An automated meter of static parameters of semiconductor devices // Izmeritel'naya Tekhnika No 12, pp. 49-52 (1996) (in Russian).

7. V.T. Kremen, An automated meter of parameters of semiconductor devices // Izmeritel'naya Tekhnika No 9, pp. 31-34 (1998) (in Russian).

8. R.V. Konakova, E.A. Soloviev, O.E. Rengevich, Facilities and techniques for diagnostics of microwave diodes and transistors, in 7th Intern. Crimean Conf. "Microwave \& Telecommunication Technology", Conf. Proc., Sevastopol, Weber Co., pp. 282-283 (1997) (in Russian).

9. I.Yu. Il'in, R.V. Konakova, O.E. Rengevich, E.A. Soloviev, A measuring module and software for test rig to check FET parameters // Izvestiya Vuzov. Elektronika No 4, pp. 101-104 (1998) (in Russian).

10. O.E. Rengevich, Effect of g-radiation and microwave radiation on the SB-FET parameters, Author's Abstract of the Candidate of Tekhnical Sci. Thesis, Kiev, Institute of Semiconductor Physics of the National Academy of Sciences of Ukraine, 2001 (in Russian).

11. N.A. Charykov, M.L. Belousov, M.V. Perevozchikov, Yu.V. Oreshnikov, V.N. Danilin, T.A. Zhukova, A programmable computer complex for determination of contact resistance to gallium nitride and related materials, in Abstracts of the AllRussia Conf. "Gallium, indium and aluminum nitrides - structures and devices", Moscow, 1-2 November 2001, p. 17 (in Russian).

12. F. Draghici, F. Mitu, G. Dilimot, I. Enache, Method to increase the thermal stability of the heating circuits, in CAS'98 Proc. (1998 Int. Semicond. Conf., Sinaia, Romania), 2, pp. 501-504 (1998).

13. V.T. Kremen, A wide-range temperature regulating device with automatic setting for regulated object // Pribory $i$ Tekhnika Eksperimenta No 5, pp. 158-160 (1998) (in Russian).

14. O.E. Rengevich, Radiation effects in HEMTs // Pis'ma v ZhTF 25(25), pp. 55-58 (1999) (in Russian). 